\title{
Effects of intravitreal injection of a Rho-GTPase inhibitor (BA-210), or \\ CNTF combined with an analogue of cAMP, on the dendritic morphology of regenerating retinal ganglion cells
}

\author{
*Eleanor S Drummond ${ }^{1,4}$, *Jennifer Rodger ${ }^{2,3}$, Marissa Penrose ${ }^{2,3}$, Donald \\ Robertson $^{1}$, Ying $\mathrm{Hu}^{1,5}$, Alan R Harvey ${ }^{1,2}$ \\ * denotes equal contribution \\ ${ }^{1}$ School of Anatomy, Physiology and Human Biology, ${ }^{2}$ Experimental and Regenerative \\ Neurosciences, ${ }^{3}$ School of Animal Biology, The University of Western Australia, WA 6009. \\ ${ }^{4}$ Present address: Department of Neuropathology, New York University, NY, USA \\ ${ }^{5}$ Present address: Regeneron Pharmaceuticals, Tarrytown, NY, USA
}

Corresponding author: Professor Alan R Harvey, School of Anatomy, Physiology and Human Biology, The University of Western Australia, 35 Stirling Highway, Crawley, WA 6009. Tel: $61864883294 \quad$ Fax: 61864881051 email: alan.harvey@uwa.edu.au

Running title: Dendritic morphology in regenerating retinal ganglion cells

Keywords: Optic nerve injury, retina, regeneration, C3 transferase, peripheral nerve graft 


\section{Abstract}

Purpose: In adult rats, intravitreal injections of the Rho-GTPase inhibitor C3 transferase (BA210), or a cocktail of recombinant ciliary neurotrophic factor (CNTF) and a cyclic AMP analogue (CPTcAMP), increase retinal ganglion cell (RGC) survival and axonal regeneration. Here we examined whether these treatments also affect the dendritic architecture of regrowing RGCs. Methods: In Fischer F344 rats, one optic nerve was cut and an autologous peripheral nerve graft was sutured onto it. Rats then received intravitreal injections $(4 \mu \mathrm{l})$ of saline, BA210 or CNTF+CPTcAMP four and eleven days after the PN graft surgery. After 5 weeks, regenerating RGCs were retrogradely labelled with fluorogold (FG) and in living wholemounts the dendritic trees of FG labelled RGCs were visualised by intracellular injection of $2 \%$ Lucifer Yellow. Results: Injection of BA-210 or CNTF+CPTcAMP resulted in significantly more regenerating RGCs with abnormal dendritic morphologies, including abnormally long looping processes. Compared to saline-injected regenerating controls, RGCs in BA-210 injected eyes had significantly smaller dendritic field areas and sparser dendrites, while in $\mathrm{CNTF}+\mathrm{CPT}$ AMP injected eyes there was increased branching of more distal dendrites. Conclusions: While both intraocular treatments enhance RGC axonal regrowth, they also induce significant changes in RGC dendritic morphology. It remains to be determined if such changes alter the function of the regenerating neuronal population. 


\section{Introduction}

Neurons in the CNS have limited ability to regenerate following injury. Various therapeutic strategies have been developed to enhance regeneration, with many studies using the rodent optic nerve (ON) as a model of CNS trauma. This is because the ON is a discrete, readily accessible CNS tract and retinal ganglion cells (RGCs) can be directly targeted by relatively non-invasive intravitreal injections that deliver therapeutic factors of known volume and dose.

The combination of transplant and pharmacological strategies has been previously shown to result in enhanced regeneration after $\mathrm{ON}$ injury. Attachment of a segment of autologous peripheral nerve $(\mathrm{PN})$ onto the transected $\mathrm{ON}$ creates a more permissive environment for the regeneration of axons and reduces glial scarring, and as a result significantly increases the number of RGC axons that regenerate long distances (eg Harvey et al., 2006). Intravitreal injection of C3 transferase, a powerful inhibitor of the Rho-GTPases A, B and C (Vogelsgesang et al., 2007; Winton et al., 2002), or injection of a cocktail of recombinant ciliary neurotrophic factor (CNTF) and a permeable cAMP analogue [8-(4-chlorophenylthio)cAMP - CPTcAMP], have both been shown to increase RGC viability after axotomy and further enhance RGC axon regeneration into ON or through PN grafts (Bertrand et al., 2007; Cui et al., 2003; Hu et al., 2007; Lehmann et al., 1999; Park et al., 2004).

After CNS injury, expression of many extracellular matrix molecules that are inhibitory to axon regeneration is increased and many of these inhibitory molecules act via a Rho-GTPase signalling pathway (Borisoff et al., 2003; Fournier et al., 2003; Monnier et al., 2003). Peripheral myelin also contains growth inhibitory molecules, albeit at lower levels (Harvey et al., 2006; Hu et al., 2007), and inactivation of Rho-GTPases by C3 transferase enhances neuronal regeneration even in a PN environment. A cell permeant C3 transferase variant, referred to here as BA-210 (Lord-Fontaine et al., 2008), is currently in the initial stages of a 
clinical trial examining the benefits of BA-210 treatment after spinal cord injury (Fehlings et al., 2011). However, effects on the morphology of regenerating CNS neurons resulting from C3 transferase administration have not, to our knowledge, been examined in vivo. Similarly, the impact of intravitreal delivery of CNTF+CPTcAMP, a potent stimulator of RGC axonal regeneration (Cui et al., 2003; Hellström et al.,2011; Hu et al., 2007; Park et al., 2004; Park et al., 2009), on the dendritic architecture of regenerating RGCs is not known. Such studies are of importance because changes in dendritic morphology may be associated with altered afferent input to these neurons and hence in the long-term may impact on their physiological properties. In this regard, both C3 transferase and CNTF have been shown to be capable of modifying dendritic structure in vitro (Ahnert-Hilger et al., 2004; Guo et al., 1999; Threadgill et al., 1997),

In this initial study, using the established PN-ON graft model of CNS injury, we therefore set out to determine, in adult rats, whether intravitreal injection of BA-210 or CNTF+CPTcAMP affected the dendritic morphology of regenerating RGCs. The ON was transected and an autologous PN sutured onto it. About 5 weeks after surgery, regenerating RGCs were retrogradely labelled with fluorogold (FG) and in living wholemounts the dendritic trees of FG labelled RGCs were visualised by intracellular injection of Lucifer Yellow (LY). Dendritic morphologies were compared to those seen in normal adult rodents (Huxlin and Goodchild, 1997), after injury to the ON (Thanos, 1988), and in non-treated PN-ON grafted animals (Chiwitt et al., 2011; Tabata and Fukuda, 1992; Thanos and Mey, 1995). 


\section{Methods}

\section{Animals and surgery}

All animal experimentation was approved by the Animal Ethics Committee of the University of Western Australia and conformed to national NHMRC guidelines. For the PN graft surgery, female Fischer F344 rats aged 8 weeks old were anaesthetised by an intraperitoneal (i.p.) injection of a 1:1 mixture of ketamine $(100 \mathrm{mg} / \mathrm{ml})$ and xylazine $(20 \mathrm{mg} / \mathrm{ml})$ at a dose of $1 \mathrm{ml} / \mathrm{kg}$. The ON transection, PN isolation and autologous graft procedure was performed as previously described (Cui et al., 2003; Hellström et al., 2011), a 1.5cm segment of the tibial nerve being sutured onto the transected ON stump, 1-1.5mm behind the optic disk, without compromising retinal blood supply. Eye ointment containing atropine sulphate (10mg/g, Troy Ilium) was applied to protect the cornea during surgery. Rats also received a subcutaneous injection of buprenorphine $(0.02 \mathrm{mg} / \mathrm{kg}$, Temgesic; Reckitt \& Colman, Hull, UK) and intramuscular injection of Benacillin (0.3mg/kg, Troy Ilium; NSW, Australia).

Under isofluorane anaesthesia (100\% $\mathrm{O}_{2}$ flow at 2.0 litre/min; $2.5 \%$ isoflurane), rats received an intravitreal injection $(4 \mu \mathrm{l})$ of either saline (control; $n=3), \quad B A-210 \quad(n=4)$ or CNTF+CPTcAMP ( $n=5)$ four and then eleven days after the PN graft surgery (Hu et al. ,2007). Purified BA-210 $(1 \mu \mathrm{g} / 4 \mu \mathrm{l}$ injection) was provided under agreement with BioAxone Biosciences Inc. (Montreal, Quebec, Canada). The cell permeable cAMP analogue CPTcAMP (0.1mM; Sigma) and CNTF $(1.5 \mu \mathrm{g}$ recombinant protein $/ 4 \mu$ injection, PeproTech, Rehovot, Israel) were commercially purchased. Retinas from unoperated F344 rats (n=2) that did not undergo ON transection were used to check the effectiveness of the LY injection technique and to confirm baseline dendritic data on normal adult rat RGC subtypes (Huxlin and Goodchild, 1997). 
About 5 weeks (35-37 days)_ after PN graft surgery, rats were anaesthetised with ketamine/xylazine (see above) and regenerating RGCs labelled by an injection of $0.2 \mu 1$ of $4 \%$ Fluorogold (FG; Fluorochrome) into the distal end of the graft 2-4 days prior to euthanasia (Cui et al., 2003). The two unoperated, non-grafted rats were anaesthetised as above, the left ON transected and FG applied to the cut end in gel foam to label all RGCs 3 days prior to euthanasia. Rats were euthanised by an overdose of sodium pentobarbital (Lethabarb; i.p.). Whole retinas were carefully removed from eyecups in oxygenated AMES buffer, the vitreous humor removed and each retina placed RGC side up on black filter paper (Rodger et al., 2012).

\section{Single cell injections}

Retinas were placed in a slide chamber compartment that was continuously perfused with oxygenated AMES buffer throughout the cell injection procedure. Pulled glass micropipettes (tip resistance of approximately $100 \mathrm{M} \Omega$ ) were filled with Lucifer Yellow (LY; Sigma; 2\% in 0.1M Tris) by capillary action. Intracellular injections of FG positive, regenerating RGCs were performed under a fluorescent microscope attached to a low light ccd camera.

\section{Immunohistochemistry for Lucifer Yellow}

Retinas were fixed in $4 \%$ paraformaldehyde for $2 \mathrm{hrs}$, washed with phosphate buffered saline (PBS;3x10 minutes) and incubated overnight at $4{ }^{\circ} \mathrm{C}$ with anti-LY antibody (Molecular Probes; 1:500) diluted in blocking buffer (1\% Triton-X100; 1\% bovine serum albumin; PBS). Retinas were washed with PBS for 1 hour and incubated with an anti-rabbit Cy3-conjugated secondary antibody (Jackson Laboratories; 1:300 in blocking buffer) for 4.5 hours at room temperature. Retinas were washed with PBS (3x10 minutes), mounted onto a glass slide and coverslipped with Citifluor. 


\section{Morphological analysis}

Confocal images of each RGC were collected using a multiphoton confocal microscope. Images were collected at the highest magnification that allowed visualisation of the complete dendritic field of each individual cell (either 10X or 20X). Consecutive images of each cell were collected at $2 \mu \mathrm{m}$ intervals at a resolution of 1024x1024 pixels in an 8-bit grey scale using a 561nm laser. Z projections of each cell were generated using ImageJ software (free download from http://rsb.info.nih.gov/ij) and all morphology measurements were made using ImageJ. Various parameters for morphology analysis were examined, primarily following the criteria outlined in Sun et al., (2002). A total of 362 RGCs was analysed in this way; 70 normal RGCs from unoperated rats and 292 regenerating RGCs. Soma area was defined as the area of the best fitting oval around the soma. The dendritic field area was defined as the area that resulted from linking all of the outer dendrites in a convex manner. The number of primary dendrites per cell was counted and the number of terminal dendrite tips was counted as a measure of branching frequency of the dendritic field. RGCs were also classified as having abnormal morphology if they possessed one or more of the following features; individual long looping processes that extended beyond the dendritic field area, multiple axon-like processes, meandering dendrites that doubled back on themselves, tangled dendritic arbours or abnormally small dendritic field areas.

Sholl analysis was carried out on 29 normal RGCs (RGC type I only) and 297 regenerating RGCs in Image J by placing concentric circles of increasing diameter (40 $\mu \mathrm{m}$ intervals) centred on the soma of each RGC. The number of intersections of RGC dendrites with each circle was counted for all control and treatment groups. The different ' $n$ ' values in each analysis are due to variation in the quality of the images which did not permit all analyses to be carried out on all cells. 
Immunohistochemistry for BIII-tubulin

Following confocal image collection, coverslips were carefully removed by immersion in PBS and retinas then immunostained for $\beta$ III-tubulin to label all surviving RGCs using the protocol described previously (Hellström et al., 2011). The total numbers of surviving, $\beta$ III-tubulin positive RGCs and FG positive regenerating RGCs were counted to ensure that the PN graft and the pharmacological injections were successful in promoting regeneration.

\section{Statistics}

RGC counts were compared using the non-parametric Kruskall-Wallis test. Statistical analysis of RGC dendritic morphology was performed using GraphPad Prism 4. For soma size and terminal tip measurements, data were normally distributed and ANOVA was used to compare multiple groups and comparisons between individual groups were performed using the Bonferroni post-hoc test. Data for dendritic field size were not normally distributed, therefore groups were compared using the non parametric Kruskal Wallis test with post hoc Dunn multiple comparisons. Chi-square analysis was used to compare the proportion of regenerating cells with abnormal morphology between groups. Sholl analysis data were analysed by repeated measures ANOVA and post-hoc Bonferroni-Dunn tests.

\section{Results}

\section{Normal RGC dendritic morphology}

The dendritic architecture of 70 normal RGCs sampled from two unoperated F344 rats was analysed. Based on morphology, 41\% of cells were classified as type I RGCs (Fig. 1A), 30\% of cells were classified as type II RGCs (Fig. 1B) and 23\% of cells were classified as type III RGCs, with 6\% of RGCs not obviously fitting into one of the three groups. This is a 
comparable distribution to previous reports (Huxlin and Goodchild, 1997). Further subclassification based on more complex dendritic architecture parameters was not attempted (eg. Sun et al., 2002) and here we follow the nomenclature described by Huxlin and Goodchild (1997). The normal morphology observed in these RGCs confirmed the effectiveness of the intracellular LY fills. Note that we saw little indication of degeneration or altered dendritic structure as a result of axotomy and fluorogold labelling at this early time-point (cf. Vidal-Sanz et al., 1987; Villegas-Peréz et al., 1988).

\section{Regenerating RGC morphology}

The numbers of surviving and regenerating RGCs in retinas after PN graft and injection of saline ( $n=3)$, BA-210 $(n=4)$ or CNTF+CPTcAMP $(n=5)$ were determined by counting the number of $\beta$ III-tubulin positive and FG positive RGCs respectively. These counts were undertaken in order to check that the injections of BA-210 or CNTF+CPTcAMP were biologically effective in enhancing axonal regeneration (Hu et al., 2007), and thus any quantitative RGC measurements would be made in successfully injected eyes. These counts are given in Table 1. Two animals, BA-210 (1) and CNTF-CPTcAMP (5), had low BIIItubulin and FG counts strongly suggestive of sub-optimal intravitreal injections. Consistent with this, the dendrites of 11 RGCs from BA-210 (1) were morphologically significantly different from the dendrites of RGCs from the other three BA-210 injected rats (Sholl analysis, data not shown). The BA-210 (4) animal was included because of a high FG count in this retina, even though the estimated $\beta$ III-tubulin count was low due to sub-optimal immunostaining (Table 1).

Counts of surviving ( $\beta$ III-tubulin positive) and regenerating (FG labelled) RGCs were similar to previous studies (Cui et al., 2003; Park et al., 2004; Hu et al., 2007), confirming that the PN 
graft procedure and intravitreal injections of each factor were effective. For example, the mean number of viable $\beta$ III-tubulin ${ }^{+}$RGCs after two CNTF+CPTcAMP injections was 8057, of which 56\% regenerated an axon. This compares with an average of 10016 RGCs in an earlier study (Hu et al., 2007), of which 54\% regrew an axon. Three intravitreal injections of CNTF+CPTcAMP yield even higher numbers (Cui et al., 2003). On average the estimated number of regenerating FG positive RGCs after two BA-210 injections (7297) was significantly greater than saline injected PN grafted controls $(2909)(\mathrm{p}<0.05$, Table 1$)$. The proportion of surviving RGCs that regrew an axon in the BA-210s group was actually higher in the present study (62\%) compared to the earlier report (37\%) (Hu et al., 2007).

As previously described (Rodger et al., 2012; Thanos, 1988), regenerating RGCs on average had significantly larger soma and significantly fewer terminal dendrite tips compared to normal RGCs (Fig. 1C, Fig. 2A). As a result, the vast majority of regenerating RGCs resembled type I RGCs, making it difficult to classify regenerating RGCs into the three major classes based on morphology as was done for normal RGCs. Only one cell out of the 292 regenerating LY injected RGCs that were analysed possessed a morphology similar to a type II RGC, even though care was taken to ensure that the whole spectrum of RGCs was injected based on soma size. Because of this, we performed two separate analyses. First, we compared all normal RGCs to all regenerating RGCs in saline control rats to confirm changes in morphology (reduction in dendritic field size and complexity) that have been reported previously in regenerating RGCs. We then wished to compare morphology between treatment groups, however the presence of multiple RGC subtypes in the normal dataset resulted in high variability compared to the regenerating RGC datasets, in which all RGCs were type I-like. Thus for these comparisons we only included in the normal RGC group those cells that were identified as Type I based on their large soma and characteristic branching pattern (Fig 1A). 
Regenerating RGCs - general morphology

All regenerating RGCs had altered morphology compared to normal RGCs in unoperated animals, regardless of injection of saline, BA-210 or CNTF+CPTcAMP (Fig 1). In the salineinjected retinae ( $\mathrm{n}=83$ ), $15.6 \%$ of cells were classified as having one or more features of abnormal morphology. Specifically, 2.7\% of injected RGCs had multiple axon-like processes, $2.4 \%$ of cells had abnormally small dendritic field areas, $8.4 \%$ of cells had tangled, unorganised dendritic arbours and $4.8 \%$ of cells had one or more meandering dendrites that doubled back on themselves. No injected RGCs had long looping processes in the saline group.

While some regenerating RGCs treated with BA-210 ( $n=77)$ had relatively organised dendritic morphology (Fig. 1D), 31\% exhibited one or more features of grossly abnormal morphology (Fig. 1E,F) which was significantly more than in the saline control group (Chi-square; $\mathrm{p}<0.05$ ). Specifically, a greater proportion of cells treated with BA-210 were found to have long looping or axon-like processes (4\%, Fig. 1I) (Chi-square; $\mathrm{p}<0.05)$ or abnormally small dendritic field areas (Fig. 1F) (Chi-square; $\mathrm{p}<0.05)$ compared to regenerating RGCs in saline injected eyes. Similar results were obtained for RGCs in retinae injected with CNTF+CPTcAMP ( $n=132)$, in which $31 \%$ of regenerating RGCs had one or more features of abnormal morphology (Figs. $1 \mathrm{G}, \mathrm{H}, \mathrm{J})$, which was a significantly higher number than observed in saline control regenerating cells (Chi-square; $\mathrm{p}<0.05$ ). Intravitreal injection of $\mathrm{CNTF}+\mathrm{CPT}$ AMP resulted in significantly more cells with long looping processes $(4 \%$,Chi-square; $\mathrm{p}<0.05)$ and multiple axon-like processes (11\%, Fig. 1J; Chi-square; $\mathrm{p}<0.05)$ than saline control regenerating cells.

\section{Quantitative morphological analysis}

As described earlier, the cell bodies of regenerating RGCs were on average larger than the overall normal RGC population (all RGC subtypes), and they had smaller and sparser dendritic trees (Fig. 2). When analysis was restricted to include type I RGCs only ( $n=29)$, compared to 
regenerating saline RGCs ( $n=83)$, intravitreal injection of CNTF+CPTcAMP $(n=132)$ or BA210 ( $n=77)$ did not further affect soma size, the number of terminal tips (Fig. 3A) or the number of primary dendrites (not shown). However, injection of BA-210 did significantly reduce dendritic field areas compared to regenerating RGCs in saline injected rats (Fig 3A). There was no difference in dendritic field area in RGCs exposed to CNTF+CPTcAMP compared to normal RGCs or saline control regenerating RGCs (Fig. 3A).

\section{Sholl analysis}

To further characterise the morphological effects of BA-210 or CNTF+CPTcAMP injection on RGC morphology, we performed a Sholl analysis to quantify the density of dendrites with increasing distance from the soma (Fig. 2C). Normal type I RGCs (n=29) from unoperated rats were included to allow comparison with RGCs in the regenerating saline controls ( $n=72)$, BA210 injected $(n=68)$ and CNTF+CPTcAMP $(n=157)$ eyes, confirming a general loss of dendritic complexity in regenerating RGCs. However, repeated measures ANOVA between the experimental graft groups revealed significant differences $(\mathrm{p}<0.0001)$ that were dependent on the distance from the soma (significant interaction between treatment and distance, $\mathrm{p}<0.0001$ ). Bonferroni-Dunn Post Hoc tests showed that RGCs from BA-210 injected retinae had fewer intersections between 80-120 $\mu \mathrm{m}$ from the soma compared to saline control regenerating RGCs ( $\mathrm{p}<0.005$ for all distances), indicating sparser dendrites. On the other hand, RGCs in CNTF+CPTcAMP injected retinae had significantly more intersections more distally at 200 $\mu \mathrm{m}$ from the soma compared to saline control regenerating RGCs ( $\mathrm{p}=0.01)$.

\section{Discussion}

In this study we have shown that when the regeneration of RGC axons is promoted by treatment with intraocular injections of either the Rho-GTPase inhibitor BA-210 or a cocktail of CNTF+CPTcAMP, the dendritic morphology of regenerating RGCs is significantly altered 
compared to saline-injected controls. The most prominent morphological changes following retinal injection of BA-210 or CNTF+CPTcAMP was the presence of long looping processes. In addition, injection of BA-210 resulted in a reduction in the size and complexity of dendritic arbors in regenerating RGCs, while in eyes injected with CNTF+CPTcAMP there was increased branching more distally.

The changes following BA-210 injections are reminiscent of less complex dendritic arborisation seen in early development of neurons, prior to elaboration of distinct axons and dendrites (Esch et al., 2000). Perhaps the more striking morphological differences observed following BA-210 treatment compared to CNTF-CPTcAMP and saline controls reflects an increased ability of the molecule to enhance RGC de-differentiation and recapitulate developmental processes that may be associated with successful long-distance axonal regeneration. The possibility is supported by evidence that C3 and its target Rho affect the cell cycle (Rohrbeck et al., 2012) and may regulate differentiation in PC12 cells (Sebok et al., 1999). Furthermore, it has previously been shown that a select population of RGCs regenerating axons into PN grafts may elaborate a new axon from a dendrite (Cho and So, 1989, 1992; Villegas-Pérez et al., 1988), as has been shown in pioneering studies of axotomised hippocampal neurons in culture (Goslin and Banker, 1990). The increased proportion of such abnormal processes in BA-210 and CNTF+CPTcAMP treated RGCs is therefore consistent with the very high rate of survival and regeneration (Hu et al., 2007) and the potent regenerative ability of these in vivo therapies.

The proportion of RGCs with unusual dendritic features in BA-210 or CNTF+CPTcAMP injected retinae (31\%) was lower than that observed in our previous study of RGCs with long term viral overexpression of neurotrophic factors (75\% for AAV-CNTF-GFP injected retinae; Rodger et al., 2012) and may reflect the transient impact of the two injections used here. In addition, the survival time was greater in the viral study (5-8 months), and it remains to be 
determined whether injection of recombinant factors also results in significant morphological changes in the majority of RGCs over the longer term.

The consistent LY labelling of the soma and the primary dendrites of these cells, and absence of label more distally, suggests that BA-210 may cause the overall loss of RGC dendrites, consistent with a morphology of less differentiated neurons (Esch et al., 2000). Sholl analysis revealed a particular loss of complexity between $80-120 \mu \mathrm{m}$ from the soma, suggesting that primary dendrites are not affected but higher order branches are lost. The location of the changes in complexity following BA-210 injection differs from CNTF+CPTcAMP-treated regenerating RGCs, in which dendritic processes were usually increased more distally relative to both BA-210 and saline controls. CNTF may thus have preserved to some extent these more peripheral dendrites, although in a previous study a loss of complexity at $200 \mu \mathrm{m}$ from the soma was seen following long term vector-mediated overexpression of a secretable form of CNTF (Rodger et al., 2012). Perhaps the effect of exogenous CNTF on dendritic complexity depends on the delivery regime, and it is also possible that the presence of CPT-cAMP may have moderated the impact of recombinant CNTF injections on RGC dendritic architecture.

The effect of the endogenous form of BA-210, intracellular C3 transferase, on dendritic morphology has been examined in vitro with mixed findings. One study found C3 transferase expression caused a marked decrease in the number of dendrites per neuron (Threadgill et al., 1997), while another found C3 transferase expression promoted increased dendritic length and branching (Ahnert-Hilger et al., 2004). The contrasting outcomes may reflect the differential effects of C3 on members of the Rho GTPase family. Rho GTPases are low molecular weight GTP-binding proteins that act as molecular switches in various signaling pathways including those involved in regulating neuronal polarity and cytoskeletal dynamics (Burridge and Wennerberg 2004; Etienne-Manneville and Hall 2002; Iden and Collard, 2008; Jaffe and Hall 2005). C3 inhibits RhoA, B and C with high affinity but has a much weaker effect on other 
GTPases Rac and cdc42 (Iden and Collard, 2008; Vogelgesang et al., 2006). RhoA and Rac-1 have contrasting effects on neuronal cytoskeletal dynamics (Chen and Firestein, 2007; Driessens et al., 2001; Iden and Collard, 2008; Luo, 2000; Tolias et al., 2011) with RhoA activation associated with neurite retraction and Rac-1 activation with neurite extension (Luo, 2000). Furthermore, previous studies have found that Rho GTPases affect the morphology of axons and dendrites differently: Rac1 is necessary for axon specification and growth whereas RhoA and Rac1 have antagonistic inhibitory and promoting roles respectively in dendritic spine morphogenesis (Ahnert-Hilger et al., 2004; Gualdoni et al., 2007; Iden and Collard, 2008).

Axotomy has been reported to cause over-activation of RhoA (Ahmed et al., 2006), presumably contributing to the loss of dendritic complexity we and others have observed in regenerating RGCs (Rodger et al., 2012; Thanos, 1988; Thanos and Mey, 1995). Interestingly, a recent study has shown that selective activation of Rac1-Pak-JNK pathways promotes RGC survival and regeneration and prevents dendrite atrophy following optic nerve lesion in the absence of a peripheral nerve graft (Lorenzetto et al., 2013). In our study, it is possible that the higher affinity of BA-210 for RhoA over Rac1 results in a change in the balance of RhoA and Rac1 activity in favour of Rac1. The net effect would be to promote axonal growth and may explain the long axonal-like processes we observed. However, this possible mechanism would be expected to rescue dendritic complexity and this was not observed in our study, suggesting complex interactions between GTPases and their signalling pathways that remain to be elucidated (Iden and Collard., 2008).

In an earlier study, adding CPTcAMP to intravitreal CNTF injections did not increase RGC survival compared to CNTF injections alone, but the combination did significantly increase the proportion of viable RGCs that regrew an axon into a PN graft (Cui et al., 2003). This effect 
was mediated, in part, by protein kinase A and other kinase systems (Park et al., 2004); however CPTcAMP also reduces suppressor of cytokine signalling (SOCS) expression after CNTF injections, thus helping to potentiate the pro-regenerative impact of this cytokine (Park et al., 2009). Effects may also be mediated through the exchange protein activated by cAMP (Epac) signalling pathway (Peace and Shewan, 2011). cAMP is known to influence neural plasticity via a range of cellular mechanisms (Benarroch, 2013; Sakamato et al., 2011; Waltereit and Weller, 2003; Wang and Zhang, 2012;), and an influence on dendritic growth and plasticity has been reported (Fujioka et al., 2004; Penzes et al., 2011; Shelly et al., 2010; Yamada et al. 2008). Thus an additional, direct effect of altered cAMP activity on the dendritic morphology of regenerating RGCs cannot be ruled out.

In conclusion, while the dendritic morphology of regenerating RGCs was altered in salineinjected control PN-grafted rats (see also Thanos, 1988; Thanos and Mey, 1995), the successful promotion of axonal regeneration using Rho inhibitors or neurotrophic factors was associated with significantly greater changes to dendritic architecture, perhaps as a reversion towards a developmental program and change of the cell to a regenerative state. Why these changes in dendritic architecture are seen in only about one third of the regenerating RGC population remains unclear and may either relate to the number of RGCs that initiate a regenerative program, or be a differential effect on different RGC subtypes. It also remains to be determined if these changes, noticeable at 5 weeks after surgery, persist over the longer-term (Rodger et al., 2012) or if the dendritic arborisation would be affected by successful connection of regenerated axons with their targets. In this pilot study we did not connect the graft to the superior colliculus, but previous studies have found that regenerated RGCs are able to reestablish at least some functional connections with target cells (Keirstead et al., 1989; Sauvé et al., 2001) that may result in the morphological stabilisation of regenerating RGCs (Thanos and Mey, 1995). 
The observed changes in dendritic architecture likely affect the number and efficacy of intraretinal afferent inputs to regenerating RGCs, potentially altering the physiology of these cells (Ferrante et al., 2013). Whether any such dendritic changes reflect a more developmental RGC phenotype, and/or whether altered inputs are functionally useful or detrimental needs to be established (Almasieh et al., 2012). As discussed elsewhere (Rodger et al., 2012), changes in dendritic field size and/or complexity could enhance detection capabilities in retinae with reduced RGC numbers but could also reduce visual acuity. Co-delivery of other drugs/therapies might also assist in preserving RGC function (eg Almasieh et al., 2010). Nonetheless these new data do suggest that it is important to monitor dendritic morphology as well as cell survival in experimental conditions designed to promote the long-distance regeneration of axons in the injured adult CNS, and future therapies may need to influence more selectively either axonal or dendritic compartments.

\section{Acknowledgements}

We thank Lisa McKerracher of BioAxone Biosciences Inc for supplying the BA-210, and Mats Hellstrom for performing the $\beta$ III-tubulin immunohistochemistry. The research was supported by grants from the NHMRC and WA Neurotrauma Research Programme.

Author Disclosure Statement: No competing financial interests exist. 


\section{References}

Ahmed, Z., Suggate, E. L., Brown, E. R., Dent, R. G., Armstrong, S. J., Barrett, L. B., Berry, M., \& Logan, A. (2006). Schwann cell-derived factor-induced modulation of the NgR/p75NTR/EGFR axis disinhibits axon growth through CNS myelin in vivo and in vitro. Brain, 129, 1517-33.

Ahnert-Hilger, G., Holtje, M., Grosse, G., Pickert, G., Mucke, C., Nixdorf-Bergweiler, B., Boquet, P., Hofmann, F., \& Just, I. (2004). Differential effects of Rho GTPases on axonal and dendritic development in hippocampal neurones. J Neurochem, 90, 9-18.

Almasieh M., Wilson, A,M,, Morquette, B., Cueva Vargas, J. L., \& Di Polo, A. (2012). The molecular basis of retinal ganglion cell death in glaucoma. Prog Retin Eye Res. 3, 152181.

Almasieh, M., Zhou,Y., Kelly, M. E., Casanova, C., \& Di Polo A. (2010). Structural and functional neuroprotection in glaucoma: role of galantamine-mediated activation of muscarinic acetylcholine receptors. Cell Death Dis, 1, e27.

Benarroch, E. E. (2013). HCN channels: function and clinical implications. Neurology, 80, 304-310.

Bertrand, J., Di Polo, A., \& McKerracher, L. (2007). Enhanced survival and regeneration of axotomized retinal neurons by repeated delivery of cell-permeable C3-like Rho antagonists. Neurobiol Dis, 25, 65-72.

Borisoff, J. F., Chan, C. C., Hiebert, G. W., Oschipok, L., Robertson, G. S., Zamboni, R., Steeves, J. D., \& Tetzlaff, W. (2003). Suppression of Rho-kinase activity promotes axonal growth on inhibitory CNS substrates. Mol Cell Neurosci, 22, 405-416.

Burridge, K., \& Wennerberg, K. (2004). Rho and Rac take center stage. Cell, 116, 167-179.

Chen, H., \& Firestein, B. L. (2007). RhoA regulates dendrite branching in hippocampal neurons by decreasing cypin protein levels. J Neurosci, 27, 8378-8386.

Chiwitt, C., Prokosch, V., Seeger, J., \& Thanos, S. (2011). Long-term morphometric stabilization of regenerating retinal ganglion cells in the adult rat. Restor Neurol Neurosci, 29, 127-139.

Cho, E. Y., \& So, K-F. (1989). De novo formation of axon-like processes from axotomized retinal ganglion cells which exhibit long distance growth in a peripheral nerve graft in adult hamsters. Brain Res, 484, 371-377.

Cho, E. Y., \& So, K-F. (1992). Characterization of the sprouting response of axon-like processes from retinal ganglion cells after axotomy in adult hamsters: a model using intravitreal implantation of a peripheral nerve. J Neurocytol, 21, 589-603. 
Cho, K. S., So, K-F., \& Chung, S. K. (1996). Induction of axon-like processes from axotomized retinal ganglion cells of adult hamsters after intravitreal injection of sciatic nerve exudate. Neuroreport, 7, 2879-2882.

Cho, K. S., Chan, P. M., So, K-F., Yip, H. K., \& Chung, S. K. (1999). Ciliary neurotrophic factor promotes the regrowth capacity but not the survival of intraorbitally axotomized retinal ganglion cells in adult hamsters. Neuroscience, 94, 623-628.

Cui, Q., Yip, H. K., Zhao, R. C., So, K-F., \& Harvey, A. R. (2003). Intraocular elevation of cyclic AMP potentiates ciliary neurotrophic factor-induced regeneration of adult rat retinal ganglion cell axons. Mol Cell Neurosci, 22, 49-61.

Driessens, M. H., Hu, H., Nobes, C. D., Self, A., Jordens, I., Goodman, C. S., \& Hall, A. (2001). Plexin-B semaphorin receptors interact directly with active Rac and regulate the actin cytoskeleton by activating Rho. Curr Biol, 11, 339-344.

Esch, T., Lemmon, V., \& Banker, G. (2000). Differential effects of NgCAM and N-cadherin on the development of axons and dendrites by cultured hippocampal neurons. $J$ Neurocytol, 29, 215-23.

Etienne-Manneville, S., \& Hall, A. (2002). Rho GTPases in cell biology. Nature, 420, 629-635.

Fehlings, M. G., Theodore, N., Harrop, J., Maurais, G., Kuntz, C., Shaffrey, C. I., Kwon, B. K., Chapman, J., Yee, A., Tighe, A., \& McKerracher, L. (2011). A Phase I/IIa Clinical Trial of a Recombinant Rho Protein Antagonist in Acute Spinal Cord Injury. $J$ Neurotrauma, 28, 787-796.

Ferrante, M., Migliore, M., \& Ascoli, G.A. (2013). Functional impact of dendritic branch-point morphology. J Neurosci, 33, 2156-2165.

Fournier, A. E., Takizawa, B. T., \& Strittmatter S. M. (2003). Rho kinase inhibition enhances axonal regeneration in the injured CNS. J Neurosci, 23, 1416-1423.

Fujioka, T., Fujioka, A., \& Duman, R.S. (2004). Activation of cAMP signaling facilitates the morphological maturation of newborn neurons in adult hippocampus $J$ Neurosci, 24, 319-328.

Goslin, K., \& Banker, G. (1990). Rapid changes in the distribution of GAP-43 correlate with the expression of neuronal polarity during normal development and under experimental conditions. J Cell Biol, 110, 1319-1331.

Gualdoni, S., Albertinazzi, C., Corbetta, S., Valtorta, F., \& de Curtis, I. (2007). Normal levels of Rac1 are important for dendritic but not axonal development in hippocampal neurons. Biol Cell, 99, 455-464. 
Guo, X., Chandrasekaran, V., Lein, P., Kaplan, P. L., \& Higgins, D. (1999). Leukemia inhibitory factor and ciliary neurotrophic factor cause dendritic retraction in cultured rat sympathetic neurons. J Neurosci, 19:, 113-2121.

Harvey, A. R., Hu, Y., Leaver, S. G., Mellough, C. B., Park, K., Verhaagen, J., Plant, G. W., \& Cui, Q. (2006). Gene therapy and transplantation in CNS repair: the visual system. Prog Retin Eye Res, 25, 449-489.

Hellström, M., Muhling, J., Ehlert, E. M., Verhaagen, J., Pollett, M. A., Hu, Y., \& Harvey, A. R. (2011). Negative impact of rAAV2 mediated expression of SOCS3 on the regeneration of adult retinal ganglion cell axons. Mol Cell Neurosci, 46, 507-515.

Hu, Y., Cui, Q., \& Harvey, A. R. (2007). Interactive effects of C3, cyclic AMP and ciliary neurotrophic factor on adult retinal ganglion cell survival and axonal regeneration. Mol Cell Neurosci, 34, 88-98.

Huxlin, K. R., \& Goodchild, A. K. (1997). Retinal ganglion cells in the albino rat: revised morphological classification. J Comp Neurol, 385, 309-323.

Iden, S., \& Collard, J. G. (2008). Crosstalk between small GTPases and polarity proteins in cell polarization. Nat Rev Mol Cell Biol, 9, 846-859.

Jaffe, A.B., \& Hall, A. (2005). Rho GTPases: biochemistry and biology. Annu Rev Cell Dev Biol, 21, 247-269.

Keirstead, S. A., Rasminsky, M., Fukuda, Y., Carter, D. A., Aguayo, A. J, \& Vidal-Sanz, M. (1989). Electrophysiological responses in hamster superior colliculus evoked by regenerating retinal axons. Science, 246, 255-257.

Kong, J-H., Fish, D. R., Rockhill, R. L., \& Masland, R. (2005). Diversity of ganglion cells in the mouse retina: unsupervised morphological classification and its limits. J Comp Neurol, 489 , 293-310.

Lehmann, M., Fournier, A., Selles-Navarro, I., Dergham, P., Sebok, A., Leclerc, N., Tigyi, G., \& McKerracher, L. (1999). Inactivation of Rho signaling pathway promotes CNS axon regeneration. J Neurosci, 19, 7537-7547.

Liu, K., Tedeschi, A., Park, K. K., \& He, Z. (2011). Neuronal intrinsic mechanisms of axon regeneration. Annu Rev Neurosci, 34, 131-152.

Lord-Fontaine, S., Yang, F., Diep, Q., Dergham, P., Munzer, S., Tremblay, P., \& McKerracher, L. (2008). Local inhibition of Rho signaling by cell-permeable recombinant protein BA-210 prevents secondary damage and promotes functional recovery following acute spinal cord injury. J Neurotrauma, 25, 1309-1322. 
Lorenzetto, E., Ettorre, M., Pontelli, V., Bolomini-Vittori, M., Bolognin, S., Zorzan, S., Laudanna, C., \& Buffelli, M. (2013). Rac1 selective activation improves retina ganglion cell survival and regeneration. PLoS One, 8, e64350

Luo, L., (2000). Rho GTPases in neuronal morphogenesis. Nat Rev Neurosci, 1, 173-180.

Monnier, P. P., Sierra, A., Schwab, J. M., Henke-Fahle, S., \& Mueller, B. K. (2003). The Rho/ROCK pathway mediates neurite growth-inhibitory activity associated with the chondroitin sulfate proteoglycans of the CNS glial scar. Mol Cell Neurosci, 22, 319330.

Murakoshi, H., Wang, H., \& Yasuda, R. (2011). Local, persistent activation of Rho GTPases during plasticity of single dendritic spines. Nature, 472, 100-104.

Park, K. K., Hu, Y., Muhling, J., Pollett, M. A., Dallimore, E. J., Turnley, A. M., Cui, Q., \& Harvey, A. R. (2009). Cytokine-induced SOCS expression is inhibited by cAMP analogue: impact on regeneration in injured retina. Mol Cell Neurosci, 41, 313-324.

Park, K., Luo, J. M., Hisheh, S., Harvey, A. R., \& Cui, Q. (2004). Cellular mechanisms associated with spontaneous and ciliary neurotrophic factor-cAMP-induced survival and axonal regeneration of adult retinal ganglion cells. J Neurosci, 24, 10806-10815.

Peace, A.G., \& Shewan, D.A. (2011). New perspectives in cyclic AMP-mediated axon growth and guidance: The emerging epoch of Epac. Brain Res Bull, 84, 280-288.

Penzes, P., Woolfrey, K. M., \& Srivastava, D. P. (2011). Epac-2-mediated dendritic spine remodelling: Implications for disease. Mol Cell Neurosci, 46, 368-380.

Rodger, J., Drummond, E. S., Hellström, M., Robertson, D., \& Harvey, A. R. (2012). Longterm gene therapy causes transgene-specific changes in the morphology of regenerating retinal ganglion cells. PLoS ONE, 7(2), e31061.

Rohrbeck, A., Kolbe, T., Hagemann, S., Genth, H., \& Just, I. (2012). Distinct biological activities of C3 and ADP-ribosyltransferase-deficient C3-E174Q. FEBS J, 279, 26572671.

Sakamoto, K., Karelina, K., \& Obrietan, K. (2011). CREB: a multifaceted regulator of neuronal plasticity and protection. $J$ Neurochem, 116, 1-9.

Sauvé, Y., Sawai, H., \& Rasminsky, M. (2001). Topological specificty in reinnervation of eth superior colliculus by regenerated retinal ganglion cell axons in adult hamsters. $J$ Neurosci, 21 951-960.

Sebök, A., Nusser, N., Debreceni, B., Guo, Z., Santos, M. F., Szeberenyi, J., \& Tigyi, G. (1999). Different roles for RhoA during neurite initiation, elongation, and regeneration in PC12 cells. J Neurochem, 73, 949-960. 
Shelly, M., Lim, B. K., Cancedda, L., Heilshorn, S. C., Gao, H., \& Poo, M-M. (2010). Local and long-range reciprocal regulation of cAMP and cGMP in axon/dendrite formation. Science, 327, 547-552.

Sun, W., Li, N., \& He, S. (2002). Large-scale morophological survey of rat retinal ganglion cells. Vis Neurosci, 19, 483-493.

Tabata, T., \& Fukuda, Y. (1992). Dendritic regrowth of retinal ganglion cells in adult rats. Neuroreport, 3, 709-712.

Thanos, S. (1988). Alterations in the morphology of ganglion cell dendrites in the adult rat retina after optic nerve transection and grafting of peripheral nerve segments. Cell Tissue Res, 254, 599-609.

Thanos, S. \& Mey, J. (1995). Type-specific stabilization and target-dependent survival of regenerating ganglion cells in the retina of adult rats. $J$ Neurosci, 15, 1057-1079.

Threadgill, R., Bobb, K., \& Ghosh, A. (1997). Regulation of dendritic growth and remodeling by Rho, Rac, and Cdc42. Neuron, 19, 625-634.

Tolias, K. F., Duman, J. G., \& Um K. (2011). Control of synapse development and plasticity by Rho GTPase regulatory proteins. Prog Neurobiol, 94, 133-148.

Vidal-Sanz, M., Bray, G. M., Villegas-Pérez, M. P., Thanos, S., \& Aguayo, A. J. (1987). Axon regeneration and synapse formation in the superior colliculus by retinal ganglion cells in the adult rat. $J$ Neurosci, 7, 2894-2909.

Villegas-Pérez, M. P., Vidal-Sanz, M., Bray, G. M., \& Aguayo, A. J. (1988). Influences of peripheral nerve grafts on the survival and regrowth of axotomized retinal ganglion cells in adult rats. $J$ Neurosci, 8, 265-280.

Vogelsgesang, M., Pautsch, A., \& Aktories, K. (2007). C3 exoenzymes, novel insights into structure and action of Rho-ADP-ribosylating toxins. Naunyn-Schmiedeberg's Arch Pharmacol, 374, 347-360.

Waltereit, R., \& Weller, M. (2003). Signaling from cAMP/PKA to MAPK and synaptic plasticity. Mol Neurobiol, 27, 99-106.

Wang, H., \& Zhang, M. (2012). The role of $\mathrm{Ca}^{2+}$-stimulated adenylyl cyclases in bidirectional synaptic plasticity and brain function. Rev Neurosci, 23, 67-78.

Winton, M. J., Dubreuil, C. I., Lasko, D., Leclerc, N., \& McKerracher, L. (2002). Characterization of new cell permeable C3-like proteins that inactivate Rho and stimulate neurite outgrowth on inhibitory substrates. J Biol Chem, 277, 32820-32829.

Yamada, R. X., Matsuki, N., \& Ikegaya, Y. (2008). cAMP differentially regulates axonal and dendritic development of dentate granule cells. J Biol Chem, 280, 38020-38028. 


\section{Figure Legends}

Fig. 1: Confocal images of RGC morphology in uninjured animals (A-B) and animals with a peripheral nerve graft (C-J). RGC morphology in uninjured animals was as previously described with cells typical of type I (A) and type II (B). Typical morphology of regenerating RGC after control injections of saline is shown in (C). The majority of regenerating RGCs had similar morphological characteristics, with large somata and reduced dendritic field complexity. Regenerating RGCs from eyes injected with BA-210 (D-F) or CNTF+CPTcAMP (G-H). Some RGCs had normal morphology after treatment with BA-210 (D) or CNTF+CPTcAMP (G), while a sub-population of cells had abnormal morphology such as disorganised dendritic arbours (E, H) and considerably reduced dendritic field areas (F).. In addition, we observed regenerating RGCs from eyes injected with BA-210 or CNTF+CPTcAMP with grossly abnormal looping processes (arrows in I) or multiple axon-like processes (arrows in J). Scale bar for all figures $=100 \mu \mathrm{m}$.

Fig. 2: Quantitative morphological measurements comparing the soma area, dendritic field area and number of terminal tips from unoperated (intact) RGCs and regenerating (saline control) RGCs. Normal RGCs from unoperated rats $(n=70)$ have smaller soma size and more terminal tips compared to regenerating RGCs from retinae injected with saline $(\mathrm{n}=83) .{ }^{*} \mathrm{p}<0.05$ compared to normal RGCs.

Fig 3: Quantitative morphological measurements comparing regenerating RGCs to only Type I RGCs in intact animals. A: morphology is compared between regenerating RGCs from intact rats (Type 1 RGCs only; $n=29)$ eyes injected with saline ( $n=83)$, BA-210 ( $n=77)$ or CNTF+CPTcAMP ( $\mathrm{n}=132)$. There was no significant difference in soma size, but dendritic 
field size and the number of terminal tips was significantly reduced in RGCs from retinae injected with BA-210. ${ }^{*} \mathrm{p}<0.05$ compared to RGCs from saline-injected retinae C: Sholl analysis shows a loss of intersections in regenerating RGC dendritic fields from retinae injected with saline $(n=72), \quad B A-210(n=68)$ and CNTF+CPTcAMP $(n=157)$ compared to

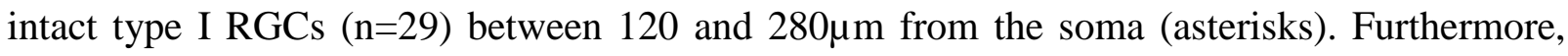
RGCs from BA-210 injected retinae have fewer intersections compared to saline injected retinae at 80 and 120mm (hash) and $\mathrm{CNTF}+\mathrm{CTP}$ AMP injected retinae have significantly more intersections compared to saline at $200 \mu \mathrm{m}$ (hat). All data are shown as mean \pm SEM,.

$\underline{\text { REVISED LEGEND - after proof review }}$

Fig. 3. Quantitative morphological measurements comparing regenerating RGCs between PNgrafted treatment groups and to Type I RGCs in intact animals. In all graphs, significant differences $(p<0.05)$ compared to intact RGCs are shown by an asterisk, and differences within regenerating RGC treatment groups are shown by a hash or a hat. A: morphology is compared between RGCs from intact rats (Type 1 RGCs only; $n=29$ ) and regenerating RGCs from eyes injected with saline $(n=83)$, BA-210 $(n=77)$ or CNTF + CPTcAMP $(n=132)$. Regenerating RGCs had larger soma size compared to intact RGCs (asterisks) but there were no significant differences between regenerating RGC treatment groups. Dendritic field size and the number of terminal tips were significantly reduced in regenerating compared to intact RGCs (asterisks) and dendritic field size was further reduced in retinae injected with BA-210 compared to RGCs from saline-injected retinae (hash). B: Sholl analysis shows a loss of intersections in regenerating RGC dendritic fields from retinae injected with saline $(n=72)$, BA-210 $(n=68)$ and CNTF + CPTcAMP $(n=157)$ compared to intact type I RGCs $(n=29)$ between 120 and $280 \mu \mathrm{m}$ from the soma (asterisks). Furthermore, RGCs from BA-210 injected retinae have fewer intersections compared to saline injected retinae at 80 and $120 \mu \mathrm{m}$ (hash) and CNTF + CTPcAMP injected retinae have significantly more intersections compared to saline at $200 \mu \mathrm{m}$ (hat). All data are shown as mean \pm SEM. 


\section{Table 1}

Estimated number of surviving ( $\beta$-III tubulin positive) RGCs and regenerating (fluorogold positive RGCs) in the 12 peripheral nerve grafted animals. Due to low counts, suggestive of less than optimal intravitreal injections, LY data from two rats (bold, asterisks) were not included in the subsequent dendritic analysis. Rat C3 (4) was included in this analysis due to high FG counts (the $\beta$-III tubulin counts were aberrantly low due to poor immunohistochemical staining in this retina). Means in parentheses exclude the two animals with sub-optimal eye injections and the estimated $\beta$-III tubulin count from C3 (4).

\begin{tabular}{|c|c|c|c|c|}
\hline Animal & $\begin{array}{l}\beta \text {-III tubulin } \\
\text { labelled RGCs }\end{array}$ & Mean & $\begin{array}{l}\text { FG labelled } \\
\text { cells }\end{array}$ & Mean \\
\hline Saline (1) & 6613 & \multirow[t]{3}{*}{6466} & 2487 & 2909 \\
\hline Saline (2) & 7115 & & 3046 & \\
\hline Saline (3) & 5671 & & 3193 & \\
\hline BA-210 (1)* & 5776* & \multirow[t]{4}{*}{8735 (11810) } & 3851* & $6435(7297)$ \\
\hline BA-210 (2) & 10502 & & 5645 & \\
\hline BA-210 (3) & 13118 & & 10196 & \\
\hline BA-210 (4) & $\begin{array}{c}5545 \text { (poor } \\
\text { immunostaining) }\end{array}$ & & 6049 & \\
\hline CNTF+CPTcAMP (1) & 7352 & \multirow[t]{5}{*}{7551 (8057) } & 3802 & 3946 (4487) \\
\hline CNTF+CPTcAMP (2) & 8717 & & 6091 & \\
\hline CNTF+CPTcAMP (3) & 8577 & & 4251 & \\
\hline CNTF+CPTcAMP (4) & 7583 & & 3802 & \\
\hline CNTF+CPTcAMP (5)* & $5524 *$ & & 1785* & \\
\hline
\end{tabular}



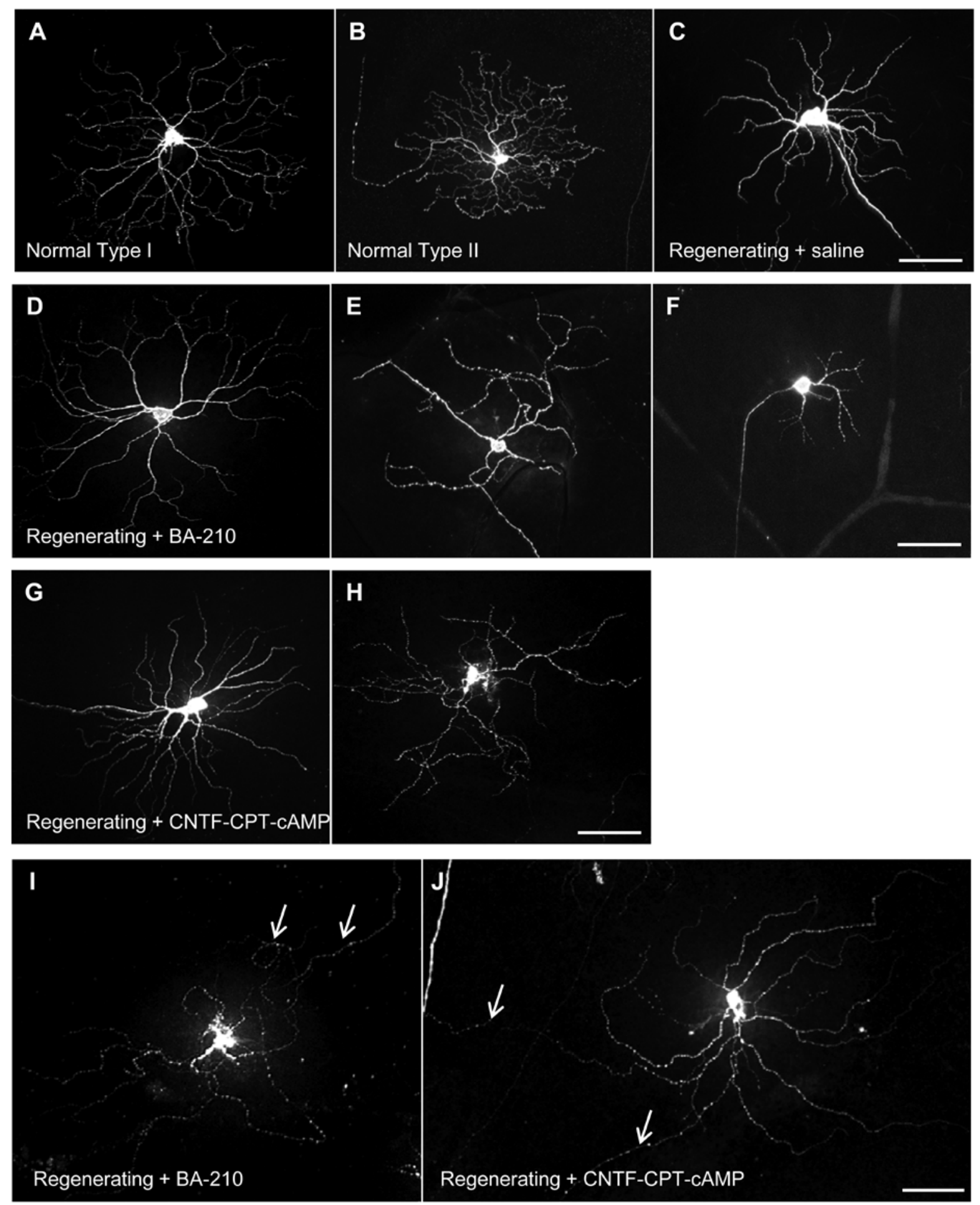

Figure 1 

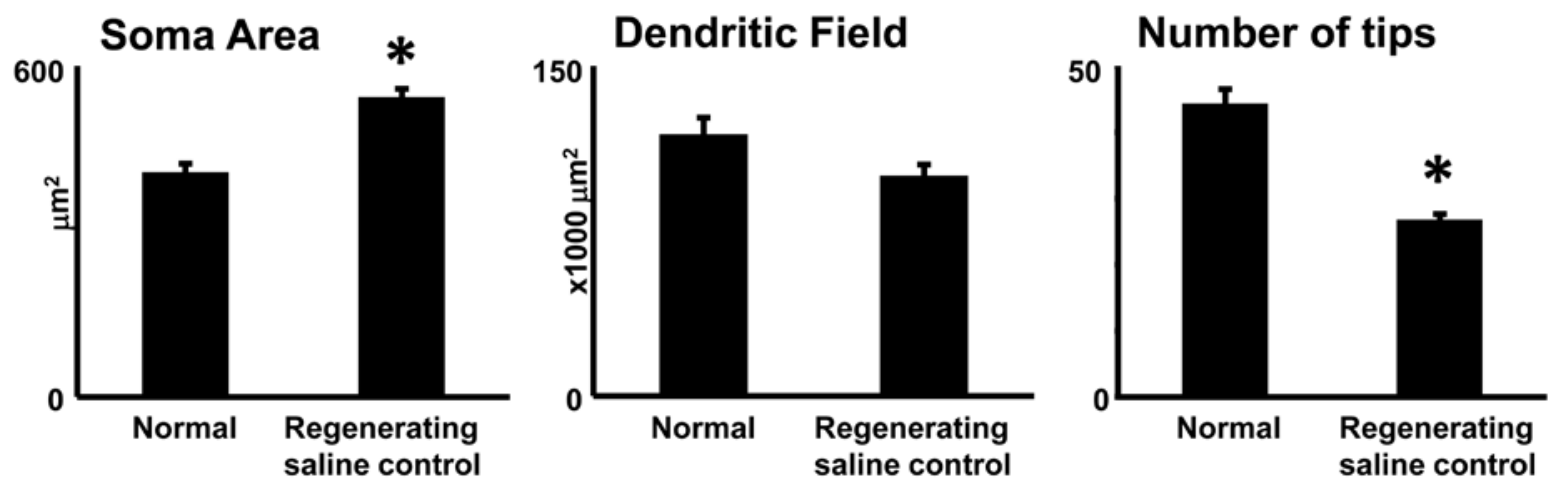

Figure 2 


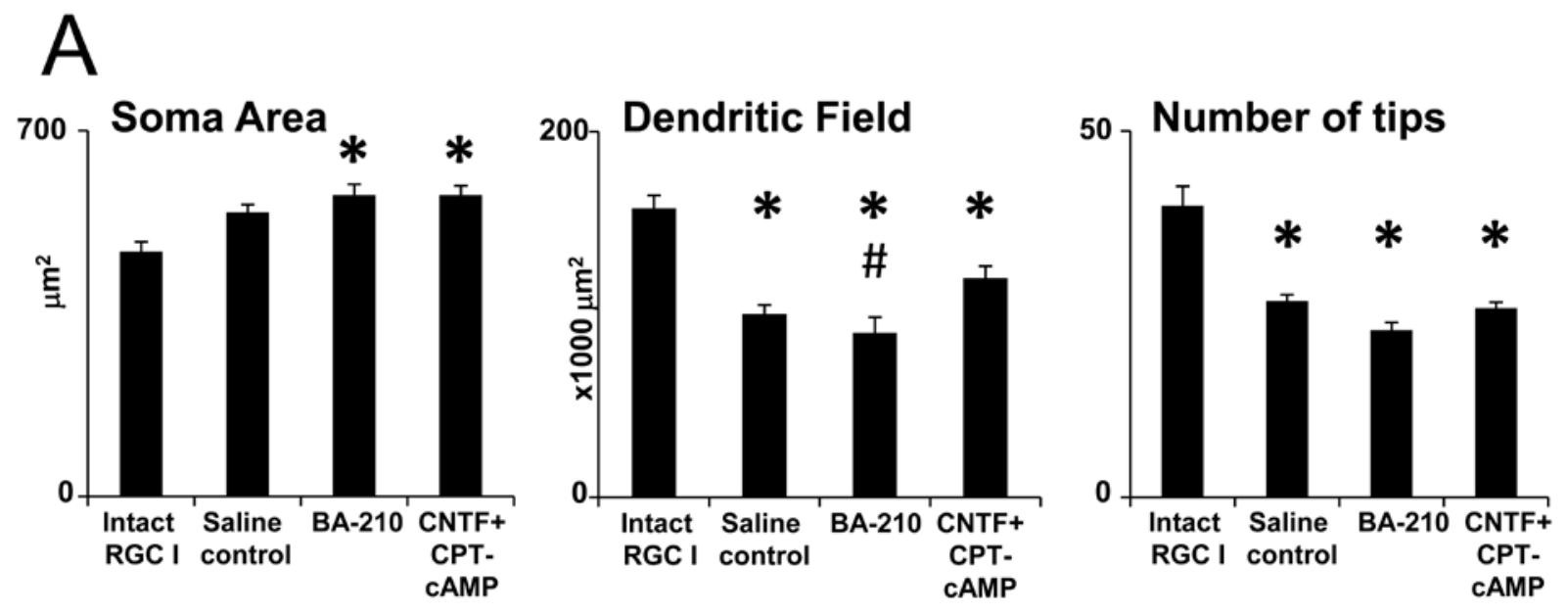

B

Sholl analysis

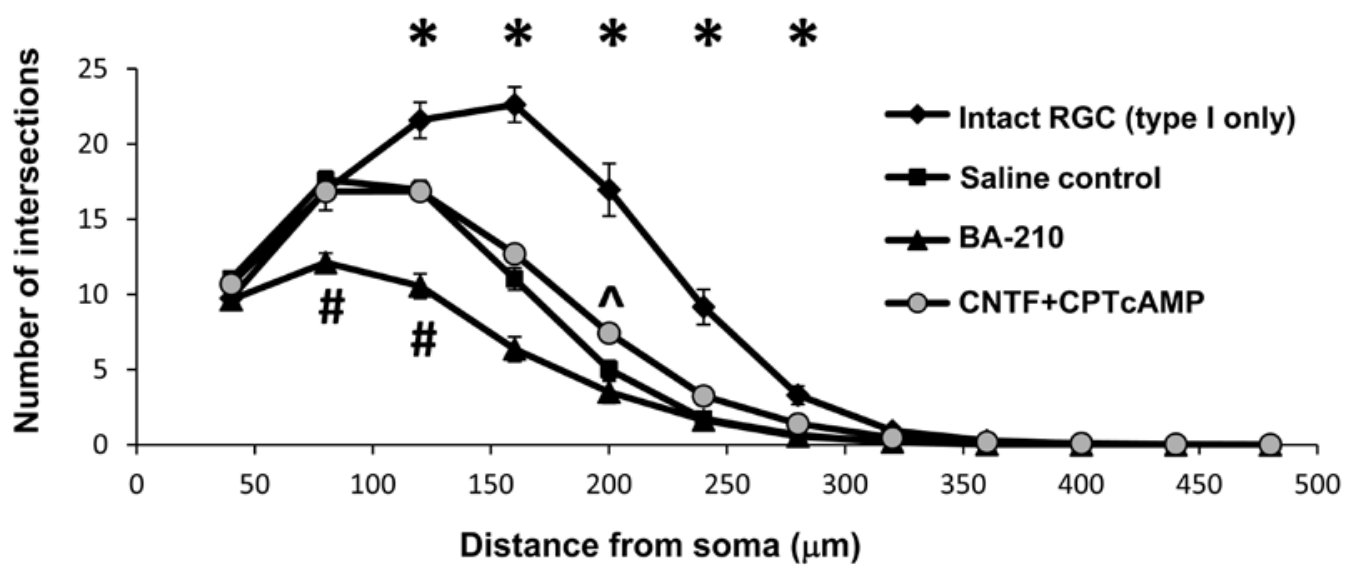

Figure 3 